\title{
Diagnostic reliability of the Berlin classification for complex MCA aneurysms - usability in a series of only giant aneurysms
}

\author{
Lars Wessels $^{1}$ - Lucius Samo Fekonja ${ }^{1}$. Johannes Achberger ${ }^{1}$. Julius Dengler ${ }^{1,2,3} \cdot$ Marcus Czabanka $^{1} \cdot$ Nils Hecht $^{1}$. \\ Ulf Schneider $^{1}$ • Dimitri Tkatschenko ${ }^{1}$ Karl-Michael Schebesch ${ }^{4}$. Nils Ole Schmidt ${ }^{4,5}$. Dorothee Mielke ${ }^{6}$. \\ Henning Hosch ${ }^{7}$. Oliver Ganslandt ${ }^{8}$. Alexander Gräwe ${ }^{9} \cdot$ Bujung Hong $^{10} \cdot$ Jan Walter ${ }^{11,12}$. Erdem Güresir ${ }^{13}$. \\ Philippe Bijlenga ${ }^{14}$. Julien Haemmerli ${ }^{1,14}$. Nicolai Maldaner ${ }^{15}$. Serge Marbacher ${ }^{16}$. Ville Nurminen ${ }^{17}$. \\ Hynek Zitek $^{18} \cdot$ Ruben Dammers $^{19} \cdot$ Naoki Kato $^{20} \cdot$ Italo Linfante ${ }^{21} \cdot$ Maria-Teresa Pedro $^{22} \cdot$ Karsten Wrede $^{23}$. \\ Wei-Te Wang ${ }^{24}$ - Maria Wostrack ${ }^{25}$. Peter Vajkoczy ${ }^{1}$ (I)
}

Received: 21 March 2020 / Accepted: 2 September 2020 / Published online: 15 September 2020

(C) The Author(s) 2020

\begin{abstract}
Background and objective The main challenge of bypass surgery of complex MCA aneurysms is not the selection of the bypass type but the initial decision-making of how to exclude the affected vessel segment from circulation. To this end, we have previously proposed a classification for complex MCA aneurysms based on the preoperative angiography. The current study aimed to validate this new classification and assess its diagnostic reliability using the giant aneurysm registry as an independent data set.

Methods We reviewed the pretreatment neuroimaging of 51 patients with giant $(>2.5 \mathrm{~cm})$ MCA aneurysms from 18 centers, prospectively entered into the international giant aneurysm registry. We classified the aneurysms according to our previously proposed Berlin classification for complex MCA aneurysms. To test for interrater diagnostic reliability, the data set was reviewed by four independent observers.

Results We were able to classify all 51 aneurysms according to the Berlin classification for complex MCA aneurysms. Eight percent of the aneurysm were classified as type $1 \mathrm{a}, 14 \%$ as type $1 \mathrm{~b}, 14 \%$ as type $2 \mathrm{a}, 24 \%$ as type $2 \mathrm{~b}, 33 \%$ as type $2 \mathrm{c}$, and $8 \%$ as type 3. The interrater reliability was moderate with Fleiss's Kappa of 0.419.

Conclusion The recently published Berlin classification for complex MCA aneurysms showed diagnostic reliability, independent of the observer when applied to the MCA aneurysms of the international giant aneurysm registry.
\end{abstract}

Keywords Giant aneurysm · Cerebral bypass $\cdot$ MCA aneurysm

\section{Introduction}

Besides improvements in endovascular techniques and versatile clipping strategies, there are still some complex aneurysms - giant, fusiform, or partially thrombosed/calcifiedwith a need for vessel sacrifice after revascularization of the downstream vessel segment [10, 13, 14, 19, 23]. Complex aneurysms of the middle cerebral artery (MCA) are a special challenge. This is due to multiple perforators in the M1

This article is part of the Topical Collection on Vascular Neurosurgery Aneurysm

Peter Vajkoczy

peter.vajkoczy@charite.de

Extended author information available on the last page of the article segment and the difficult accessibility of M1 and M2 branches, especially in very large aneurysms [6].

For the preoperative planning of cerebral revascularization and aneurysm occlusion, it is of importance to anticipate the intraoperative anatomy. Besides new developments in the field of 3D reconstruction CT angiography, digital subtraction angiography remains the best solution to estimate the orientation of the aneurysm to the vessel branches $[1,8]$.

The preoperative planning has to address two questions. The first question aims at determining the strategy of how to handle the aneurysm. Ideally, the goal should be to trap the aneurysm, but sometimes, this is not possible without placing the patient at risk of perforator ischemia or due to inaccessibility of the inflow or outflow segments. In cases where trapping is not an option, fallback strategies are proximal or distal occlusion. The second question to answer is what kind of 
revascularization is necessary, depending on the strategy chosen for aneurysm occlusion.

The pertinent literature tries to propose a clear recommendation for the technique to use for revascularization and aneurysm occlusion [20]. But since there is a large armamentarium for surgical strategies, there is no ideal solution. From our point of view, any recommendation should therefore primarily focus on the strategies on how to expose the aneurysm as well as the inflow and outflow segments, how to handle the aneurysm, and how to avoid complications.

Due to the lack of a classification addressing these aspects, we have proposed a classification for complex MCA aneurysms. We classified complex MCA aneurysms according to their localization into six categories: M1 aneurysms (type 1) subdivided into 1a without intra-aneurysmatic thrombosis and $1 \mathrm{~b}$ with preexisting intra-aneurysmatic thrombosis, M1/M2 bifurcational aneurysms (type 2) subdivided into $2 \mathrm{a}$ with the M2 branches underneath the aneurysm in the ap view with good accessibility of the M2 branches, $2 \mathrm{~b}$ where the aneurysm divides the M2 branches apart, and 2c where the M2 branches are hidden behind the aneurysm. Type 3 aneurysms are postbifurcational aneurysms without the involvement of the bifurcation (Fig. 1) [21]. Our series included 50 patients with complex aneurysms from our institution, in a retrospective monocentric design. To further validate our concept, this study now aimed at validating the Berlin classification for complex MCA aneurysms using an independent data set of patients with giant intracranial aneurysms of the MCA and determine its diagnostic reliability and interobserver applicability.

a

b

C

1
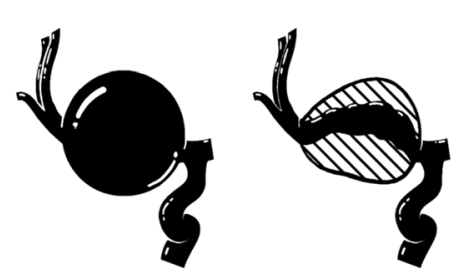

2
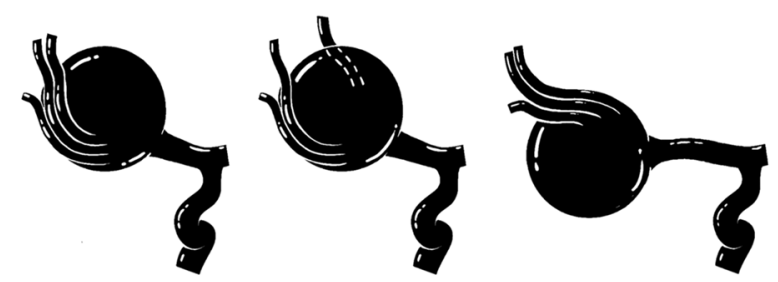

3

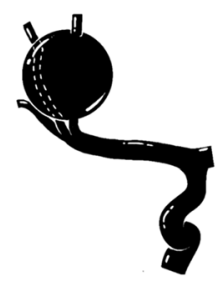

\section{Methods}

We retrospectively reviewed 51 cases with giant MCA aneurysms from 18 centers participating in the international giant aneurysm registry [4]. We classified the aneurysms based on the ap view of the pretreatment DSA as previously reported. For testing the interrater reliability, four independent observers classified the 51 aneurysms according to our previously reported classification [21]. All observers were blinded for the clinical course of the patients and possible treatment. The observers had different levels of experience: rater 3 and 4 are vascular experienced neurosurgeons, and rater 1 and 2 are neurosurgical residents with vascular interest. We analyzed interrater reliability using the Fleiss's Kappa; agreement was graded according to the Landis-Koch criteria $(<0$ poor agreement, $0.0-0.20$ slight agreement, $0.21-0.40$ fair agreement, $0.41-0.60$ moderate agreement, $0.61-0.80$ substantial agreement, 0.81-1.0 almost perfect agreement) [15].

\section{Results}

The data set provided by the members of the international giant aneurysm registry consisted of 51 patients with giant aneurysms of the MCA. We were able to classify all aneurysms according to our preexisting classification system. Examples of the different types of aneurysms are given in Fig. 2.

Four (8\%) aneurysms were classified as type 1a, $7(14 \%)$ as type $1 \mathrm{~b}, 7(14 \%)$ as type 2a, $12(24 \%)$ as type $2 \mathrm{~b}, 17(33 \%)$ as type $2 \mathrm{c}$, and $4(8 \%)$ as type 3 . As Fig. 3 shows, the results differ from our previously published institutional series (1a: $6 \%, 1 \mathrm{~b}: 4 \%, 2 \mathrm{a}: 16 \%, 2 \mathrm{~b}: 16 \%, 2 \mathrm{c}: 28 \%, 3: 30 \%)$.

The interrater reliability was moderate with $0.419(95 \% \mathrm{CI}$ $0.418-0.42$ ), and the different rater only agreed in 9 cases (Fig. 4).

None of the independent observers reported any difficulties applying the classification to the image set.

\section{Discussion}

In this study, we demonstrate that the algorithmic structure can be applied to a separate series of 51 giant MCA aneurysms. The interrater reliability of 0.49 was moderate, and there was a large disagreement by grading the different aneurysm which might be due to the complexity of the aneurysms, since there does not seem to be an association with the level of experience. Since the observer in this study was restricted to AP and lateral view of the DSA, the disagreement can also be due to the need for detailed views and $3 \mathrm{D}$ reconstructions to evaluate such complex lesions. The distribution of the different types of MCA aneurysms differed from our previously

Fig. 1 Overview of the classification for MCA aneurysm 
Fig. 2 Examples for each type of aneurysm out of the giant aneurysm registry set of cases a
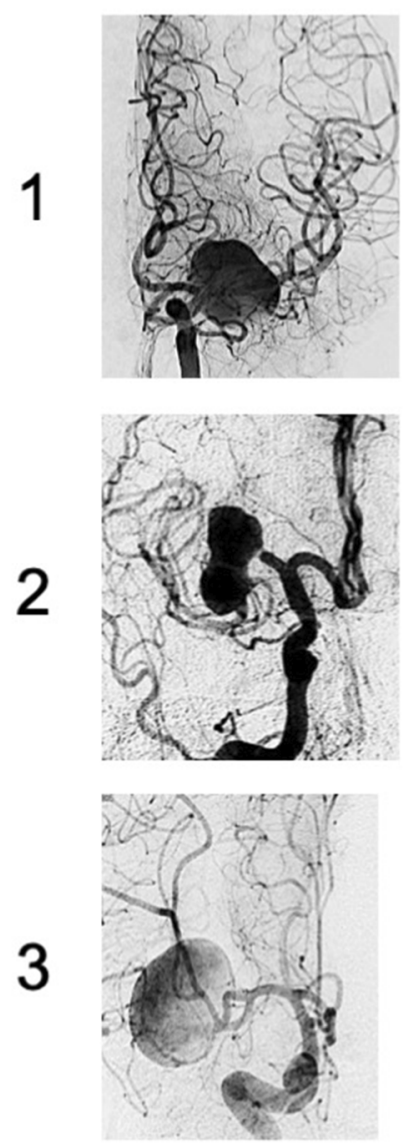

b
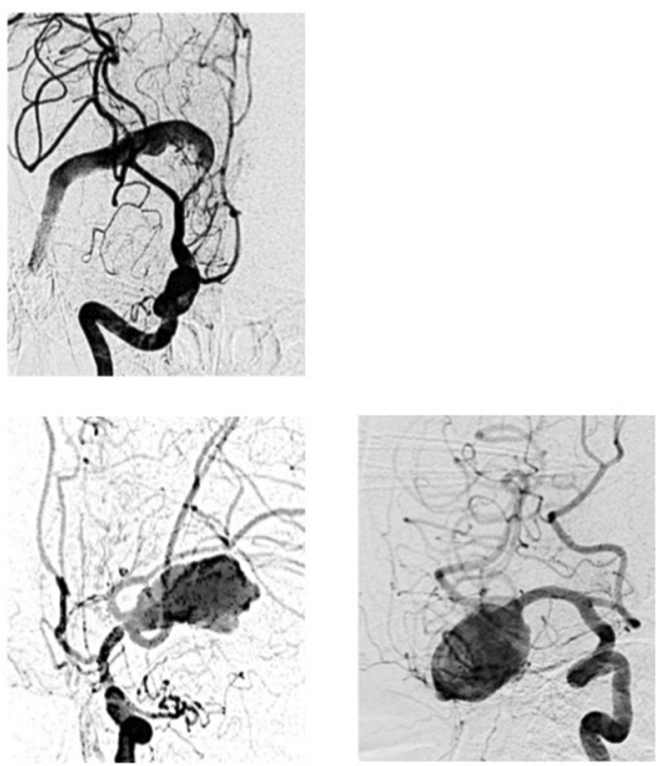

reported series. One of the main reasons for this could be that in the previously reported series, not all aneurysms (56\%) were giant, since we included all patients in whom an MCA aneurysm is treated with revascularization. Taking the MCA branching anatomy into account, type 3 aneurysms are limited in size, and the relatively low flow $[3,22]$ in the more distal MCA might not support the aneurysm growth to the same extent as in the more proximal segments. In general, the larger the size of the aneurysm, the greater is the chance that the

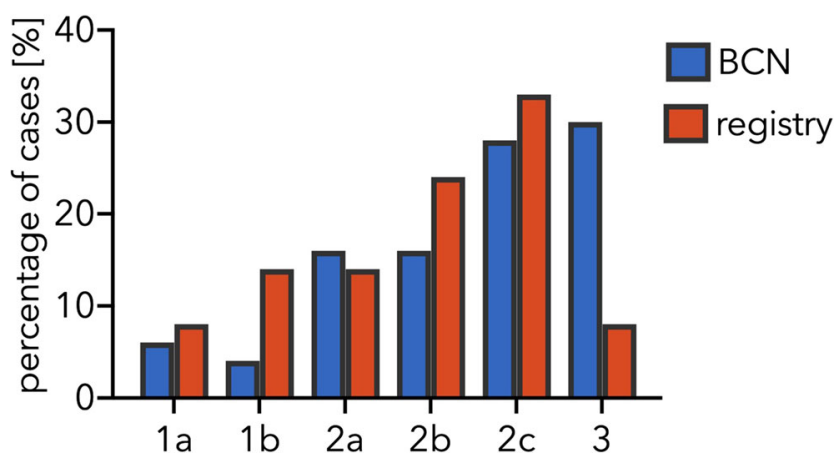

Fig. 3 Percentage of cases for each type of aneurysm compared between the previously reported series of the Neurosurgical Department of the Charité University Medicine Berlin (BCN) compared with the series of cases provided by the giant aneurysm registry bifurcation is involved, which is in line with previous reports on the distribution of giant aneurysms affecting the MCA [16].

The rupture rate of aneurysms increases with size $[9,12]$. For untreated giant intracranial aneurysms, the 1-year rupture rate is $25.3 \%$ in non-cavernous intracranial aneurysms with a case fatality of $100 \%$ [5]. This underlines the need for treatment of these lesions. Our classification aims at improving the preoperative planning with an algorithmic approach to anticipate the relationship between the aneurysm, the M2 branches, and the incorporated perforators. Further, our classification focuses on predicting the accessibility of the inflow and outflow segments. Our classification system complements other reports focusing on the bypass technique at the pre-bifurcation, bifurcation, and postbifurcation levels [20].

In our classification system, M1 aneurysms are divided into aneurysms with (type 1b) and without (1a) intra-aneurysmatic thrombosis. The giant aneurysm cohort has more cases with intra-aneurysmatic thrombosis due to the large size. This categorization is important because in $1 \mathrm{~b}$ aneurysms, the occlusion strategy can be more aggressive since perforators are likely already occluded, whereas in 1a aneurysms, complete trapping would cause infarction in the internal capsule. Since to the best of our knowledge the current literature does not 
Fig. 4 Rating of every aneurysm by the different observers
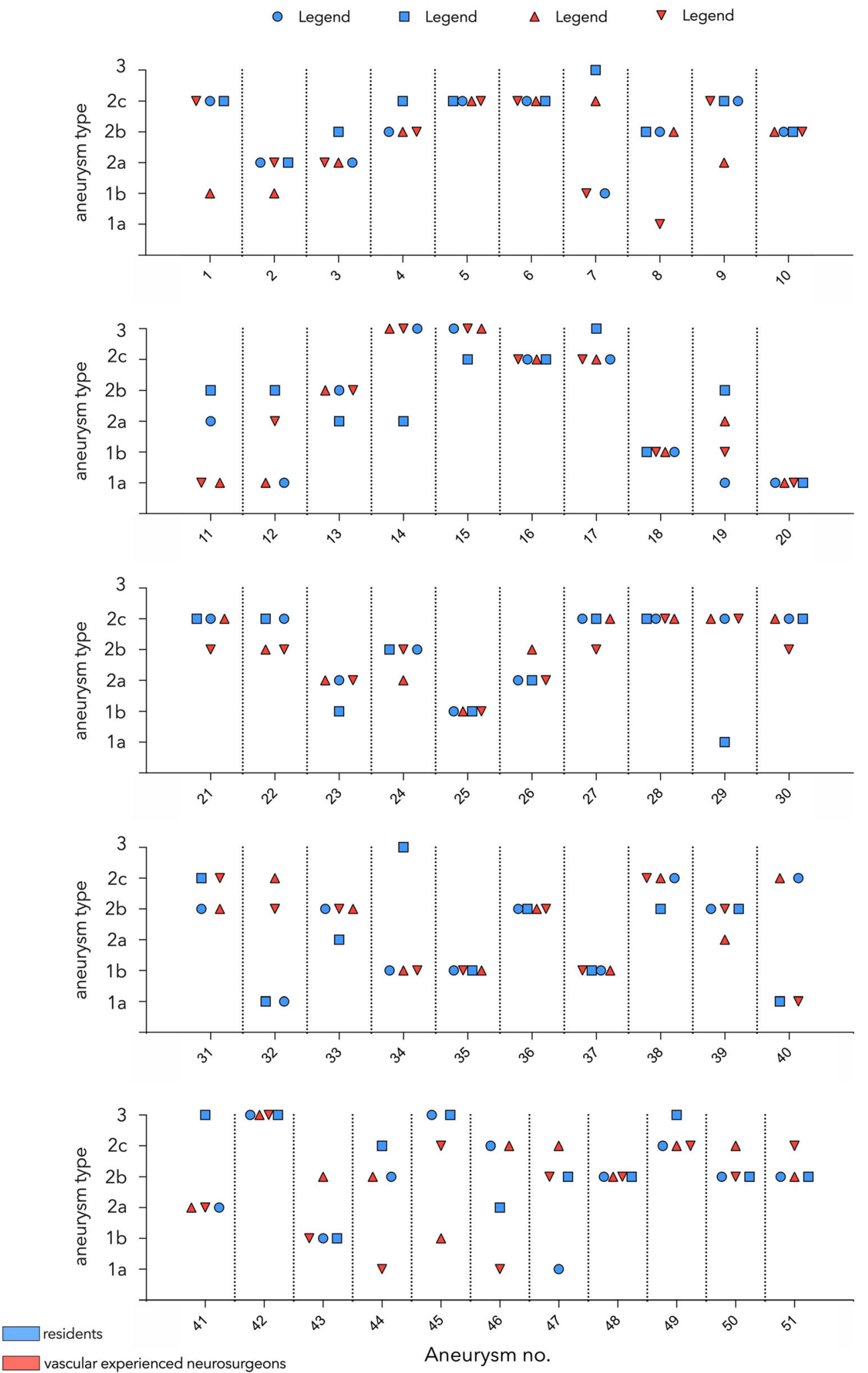

provide information about the risk of complete trapping partial thrombosed M1 aneurysm, we recommend electrophysiological monitoring and test occlusion in all cases before trapping an MCA aneurysm. The categorization of the aneurysms affecting the bifurcation into type 2a-c helps estimating the chances of complete revascularization of the outflow segment, which the patient needs to be informed about and is essential for planning and successful trapping [21]. 
Although intraoperative techniques like indocyanine green videoangiography, flowmeter, or non-quantitative microdoppler allow assessment of blood flow in the recipient $[1,2,7,18]$, the decision of what kind of revascularization and aneurysm occlusion strategy should be used is not possible with these techniques. Further, it is of high importance to be able to perform the surgical planning beforehand, in order to counsel not only the patient but also the OR team about the planned surgical steps. Despite emerging 3D reconstruction techniques of CT angiography, and MR-based flow modeling, classical digital subtraction angiography still provides the most crucial information on deciding which treatment is best for complex aneurysms $[8,11,17]$. Nevertheless, 3D reconstruction techniques can help understanding the anatomy of the aneurysm.

Importantly, the structured classification that we propose serves as a simple, reliable, and cost-effective preoperative planning tool, which might help anticipate potential intraoperative pitfalls and facilitate the development of alternative surgical strategies before, instead of during the procedure.

Funding Open Access funding enabled and organized by Projekt DEAL.

\section{Compliance with ethical standards}

Conflict of interest The authors declare that they have no conflict of interest.

Ethical approval All procedures performed in studies involving human participants were in accordance with the ethical standards of the institutional and/or national research committee and with the 1964 Helsinki declaration and its later amendments or comparable ethical standards.

For this type of study, formal consent is not required.

Open Access This article is licensed under a Creative Commons Attribution 4.0 International License, which permits use, sharing, adaptation, distribution and reproduction in any medium or format, as long as you give appropriate credit to the original author(s) and the source, provide a link to the Creative Commons licence, and indicate if changes were made. The images or other third party material in this article are included in the article's Creative Commons licence, unless indicated otherwise in a credit line to the material. If material is not included in the article's Creative Commons licence and your intended use is not permitted by statutory regulation or exceeds the permitted use, you will need to obtain permission directly from the copyright holder. To view a copy of this licence, visit http://creativecommons.org/licenses/by/4.0/.

\section{References}

1. Amin-Hanjani S, Alaraj A, Charbel FT (2010) Flow replacement bypass for aneurysms: decision-making using intraoperative blood flow measurements. Acta Neurochir 152(6):1021-1032 discussion 1032

2. Balamurugan S, Agrawal A, Kato Y, Sano H (2011) Intra operative indocyanine green video-angiography in cerebrovascular surgery: an overview with review of literature. Asian J Neurosurg 6(2):88-93
3. Blanco PJ, Müller LO, Spence JD (2017) Blood pressure gradients in cerebral arteries: a clue to pathogenesis of cerebral small vessel disease. Stroke Vasc Neurol 2(3):108-117

4. Dengler J, Heuschmann PU, Endres M, Meyer B, Rohde V, Rufenacht DA, Vajkoczy P (2011) The rationale and design of the giant intracranial aneurysm registry: a retrospective and prospective study. Int J Stroke 6(3):266-270

5. Dengler J, Rüfenacht D, Meyer B et al (2019) Giant intracranial aneurysms: natural history and 1-year case fatality after endovascular or surgical treatment. J Neurosurg:1-9. https://doi. org/10.3171/2019.8.JNS183078

6. Esposito G, Durand A, Van Doormaal T, Regli L (2012) Selectivetargeted extra-intracranial bypass surgery in complex middle cerebral artery aneurysms: correctly identifying the recipient artery using indocyanine green videoangiography. Neurosurgery 71(SUPPL.2):ons274-ons285

7. Esposito G, Regli L (2014) Selective targeted cerebral revascularization via microscope integrated indocyanine green videoangiography technology. Trends Neurovascular Interv. Springer International Publishing, Cham, pp. 59-64

8. Esposito G, Regli L, Esposito G, Regli L (2014) Surgical decisionmaking for managing complex intracranial aneurysms. Trends Neurovascular Interv. Springer Wien, Cham, pp. 3-11

9. Greving JP, Wermer MJHH, Brown RD et al (2014) Development of the PHASES score for prediction of risk of rupture of intracranial aneurysms: a pooled analysis of six prospective cohort studies. Lancet Neurol 13(1):59-66

10. Hopkins LN, Grand W (1979) Extracranial-intracranial arterial bypass in the treatment of aneurysms of the carotid and middle cerebral arteries. Neurosurgery 5(1):21-31

11. Hussein AE, Brunozzi D, Shakur SF, Ismail R, Charbel FT, Alaraj A (2018) Cerebral aneurysm size and distal intracranial hemodynamics: an assessment of flow and pulsatility index using quantitative magnetic resonance angiography. Clin Neurosurg 83(4):660 665

12. Investigators TUJ (2012) The natural course of unruptured cerebral aneurysms in a Japanese cohort. N Engl J Med 366(26):2474-2482

13. Kalani MYS, Ramey W, Albuquerque FC, McDougall CG, Nakaji P, Zabramski JM, Spetzler RF (2014) Revascularization and aneurysm surgery. Neurosurgery 74(5):482-498

14. Kalani MYS, Rangel-Castilla L, Ramey W, Nakaji P, Albuquerque FC, McDougall CG, Spetzler RF, Zabramski JM (2015) Indications and results of direct cerebral revascularization in the modern era. World Neurosurg 83(3):345-350

15. Koch GG, Landis JR, Freeman JL, Freeman DH, Lehnen RG (1977) A general methodology for the analysis of experiments with repeated measurement of categorical data. Biometrics 33(1):133

16. Nurminen V, Lehecka M, Chakrabarty A, Kivisaari R, Lehto H, Niemelä M, Hernesniemi J (2014) Anatomy and morphology of giant aneurysms - angiographic study of 125 consecutive cases. Acta Neurochir 156(1):1-10

17. Ogawa T, Okudera T, Noguchi K, Sasaki N, Inugami A, Uemura K, Yasui N (1996) Cerebral aneurysms: evaluation with threedimensional CT angiography. AJNR Am J Neuroradiol 17(3): $447-454$

18. Rustemi O, Amin-Hanjani S, Shakur SF, Du X, Charbel FT (2016) Donor selection in flow replacement bypass surgery for cerebral aneurysms: quantitative analysis of long-term native donor flow sufficiency. Neurosurgery 78(3):332-341

19. Straus DC, Brito da Silva H, McGrath L, Levitt MR, Kim LJ, Ghodke BV, Barber JK, Sekhar LN (2017) Cerebral revascularization for aneurysms in the flow-diverter era. Neurosurgery 80(5): 759-768

20. Tayebi Meybodi A, Huang W, Benet A, Kola O, Lawton MT (2017) Bypass surgery for complex middle cerebral artery 
aneurysms: an algorithmic approach to revascularization. J Neurosurg 127(3):463-479

21. Wessels L, Fekonja LS, Vajkoczy P (2019) Bypass surgery of complex middle cerebral artery aneurysms - technical aspects and outcomes. Acta Neurochir 161(10):1981-1991

22. Zarrinkoob L, Ambarki K, Wåhlin A, Birgander R, Eklund A, Malm J (2015) Blood flow distribution in cerebral arteries. J Cereb Blood Flow Metab 35(4):648-654
23. Zavanone M, Balbi S, Spetzler RF, Riina HA, Lemole GM (2001) Giant aneurysms. Neurosurgery 49(4):902-908

Publisher's note Springer Nature remains neutral with regard to jurisdictional claims in published maps and institutional affiliations.

\section{Affiliations}

Lars Wessels ${ }^{1}$ - Lucius Samo Fekonja ${ }^{1}$ - Johannes Achberger ${ }^{1}$. Julius Dengler ${ }^{1,2,3}$ - Marcus Czabanka ${ }^{1}$. Nils Hecht ${ }^{1}$. Ulf Schneider ${ }^{1}$. Dimitri Tkatschenko ${ }^{1}$. Karl-Michael Schebesch ${ }^{4} \cdot$ Nils Ole Schmidt $^{4,5}$ • Dorothee Mielke ${ }^{6}$. Henning Hosch ${ }^{7}$. Oliver Ganslandt ${ }^{8}$. Alexander Gräwe ${ }^{9} \cdot$ Bujung Hong $^{10} \cdot$ Jan Walter $^{11,12} \cdot$ Erdem Güresir $^{13}$. Philippe Bijlenga ${ }^{14}$. Julien Haemmerli ${ }^{1,14}$. Nicolai Maldaner ${ }^{15}$. Serge Marbacher ${ }^{16}$. Ville Nurminen ${ }^{17}$. Hynek Zitek ${ }^{18}$. Ruben Dammers ${ }^{19}$. Naoki Kato ${ }^{20}$ - Italo Linfante ${ }^{21}$. Maria-Teresa Pedro ${ }^{22} \cdot$ Karsten Wrede $^{23}$. Wei-Te Wang ${ }^{24}$. Maria Wostrack ${ }^{25}$ • Peter Vajkoczy ${ }^{1}$

1 Department of Neurosurgery and Center for Stroke Research Berlin (CSB), Charité Universitätsmedizin Berlin, Charitéplatz 1, 10117 Berlin, Germany Department of Neurosurgery, Helios Clinic, Bad Saarow, Germany

3 Brandenburg Medical School Theodor Fontane, Campus Bad Saarow, Germany

4 Department of Neurosurgery, University of Regensburg, Regensburg, Germany

5 Department of Neurosurgery, University Medical Center HamburgEppendorf, Hamburg, Germany

6 Department of Neurosurgery, Georg-August-University Goettingen, Göttingen, Germany

7 Department of Neurosurgery, Vivantes Klinikum im Friedrichshain, Berlin, Germany

8 Department of Neurosurgery, Klinikum Stuttgart, Germany

9 Department of Neurosurgery, Unfallkrankenhaus Berlin, Germany

10 Department of Neurosurgery, Hannover Medical School, Hannover, Germany

11 Department of Neurosurgery, University Hospital Jena, Jena, Germany

12 Department of Neurosurgery, Medical Center Saarbrücken, Saarbrücken, Germany

13 Department of Neurosurgery, University Hospital Bonn, Bonn, Germany
14 Service de Neurochirurgie, Faculté de Médecine de Genève and Hôpitaux Universitaire de Genève, Geneva, Switzerland

15 Department of Neurosurgery, University Hospital of Zurich, Zürich, Switzerland

16 Department of Neurosurgery, Kantonsspital Aarau, Aarau, Switzerland

17 Neurosurgery, University of Helsinki and Helsinki University Hospital, Helsinki, Finland

18 Department of Neurosurgery, J. E. Purkinje University, Masaryk Hospital, Ústí nad Labem, Czech Republic

19 Erasmus Stroke Center, Erasmus MC University Hospital, Rotterdam, The Netherlands

20 Department of Neurosurgery, Jikei University School of Medicine, Tokyo, Japan

21 Interventional Neuroradiology and Endovascular Neurosurgery at Miami Cardiac and Vascular Institute and Baptist Neuroscience Institute, Miami, USA

22 Department of Neurosurgery, University Hospital of Ulm, Ulm, Germany

23 Department of Neurosurgery, University of Essen, Duisburg, Germany

24 Department of Neurosurgery, Medical University, Vienna, Austria

25 Department of Neurosurgery, Technical University of Munich, Munich, Germany 